\title{
Dehiscence of patch augmentation of a left-sided atrioventricular valve related to strenuous isometric exercise: Case report and failure analysis
}

\author{
Peter E. Hammer, PhD, ${ }^{\mathrm{a}}$ Christopher W. Baird, MD, ${ }^{\mathrm{a}}$ Pedro J. del Nido, MD, ${ }^{\mathrm{a}}$ and Gerald R. Marx, MD, ${ }^{\mathrm{b}}$ \\ Boston, Mass
}

\author{
From the Departments of ${ }^{\mathrm{a} C}$ ardiac Surgery and ${ }^{\mathrm{b}}$ Cardiology, Boston Children's Hospital, Boston, Mass. \\ No funding was provided for the work. \\ Disclosures: Authors have nothing to disclose with regard to commercial support. \\ Received for publication Feb 13, 2018; revisions received April 9, 2018; accepted for publication April 24, 2018 ; \\ available ahead of print May 24, 2018. \\ Address for reprints: Peter E. Hammer, PhD, Department of Cardiac Surgery, Boston Children's Hospital, 300 \\ Longwood Ave, Boston, MA 02115 (E-mail: peter.hammer@childrens.harvard.edu). \\ J Thorac Cardiovasc Surg 2018; 156:e165-8 \\ $0022-5223 / \$ 36.00$ \\ Copyright (c) 2018 by The American Association for Thoracic Surgery \\ https://doi.org/10.1016/j.jtcvs.2018.04.101
}

Video clip is available online.

We report a case of the abrupt dehiscence of a patch augmentation of a left-sided atrioventricular valve in a 20-year-old male patient who participated in strenuous isometric exercise. Weight lifting is accompanied by a large blood pressure increase, ${ }^{1}$ and we hypothesized that the dehiscence was due to elevated loads at the suture line caused by high systolic pressures. To test this hypothesis, we calculated peak estimated load on the valve patch suture line during weight lifting and compared this load with experimental measurements of suture retention in the patch material.

\section{CLINICAL SUMMARY}

The 21-year-old male patient had undergone surgery in infancy for primum atrial septal defect and cleft left-sided atrioventricular valve. At 20 years of age, he underwent reoperation for severe regurgitation of the valve, consisting of patch augmentation of the mural leaflet with PhotoFix (CryoLife Inc, Kennesaw, Ga), bovine pericardium stabilized with a dye-mediated photo oxidation fixation process. After surgery, the patient resumed normal activity, including strenuous weight lifting, and he was well until 5 months later, when he experienced sudden onset of dyspnea and exercise intolerance. Echocardiography showed severe regurgitation emanating from the region of the mural leaflet (Figure 1, A). Three-dimensional imaging (Figure 1, B) depicted a disruption in the mural leaflet at the annulus. Intraoperative inspection revealed that the running suture had pulled through the patch at multiple sites along the annulus (Figure 1, $C$ and $D$, and Video 1).

\section{Analysis of Stress}

\section{DISCUSSION}

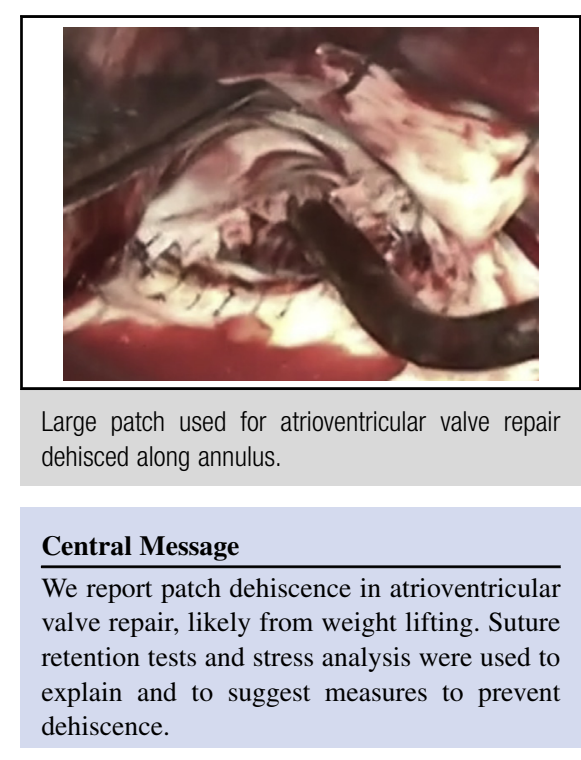

See Editorial Commentary page e 169.

To analyze the patch dehiscence, we determined the maximum expected leaflet load in a normal mitral valve at typical peak systolic pressures according to published studies. ${ }^{2}$ During weight lifting, systolic pressures as high as $480 \mathrm{~mm} \mathrm{Hg}$ have been measured in normotensive subjects. ${ }^{1}$ Leaflet stress increases in proportion to pressure, so we scaled our estimate to reflect this elevated systolic pressure, yielding an estimate of the maximum expected load on the leaflet near the annulus of $1.6 \mathrm{~N} / \mathrm{mm}$. We then measured suture retention strength, which when combined with an estimated suture spacing of $2 \mathrm{~mm}$ resulted in a suture line strength of $1.6 \mathrm{~N} / \mathrm{mm}$, indicating that the maximum expected load on the repaired valve during weight lifting might indeed reach the level at which the suture line is predicted to fail. See the Appendix for details on the calculation of loads in the valve leaflet and the suture line.

For robust design, a structure's strength should exceed its maximum working load by some safety factor, commonly calculated as the ratio of strength to maximum working load. For our reconstructed valve, we estimate a safety 


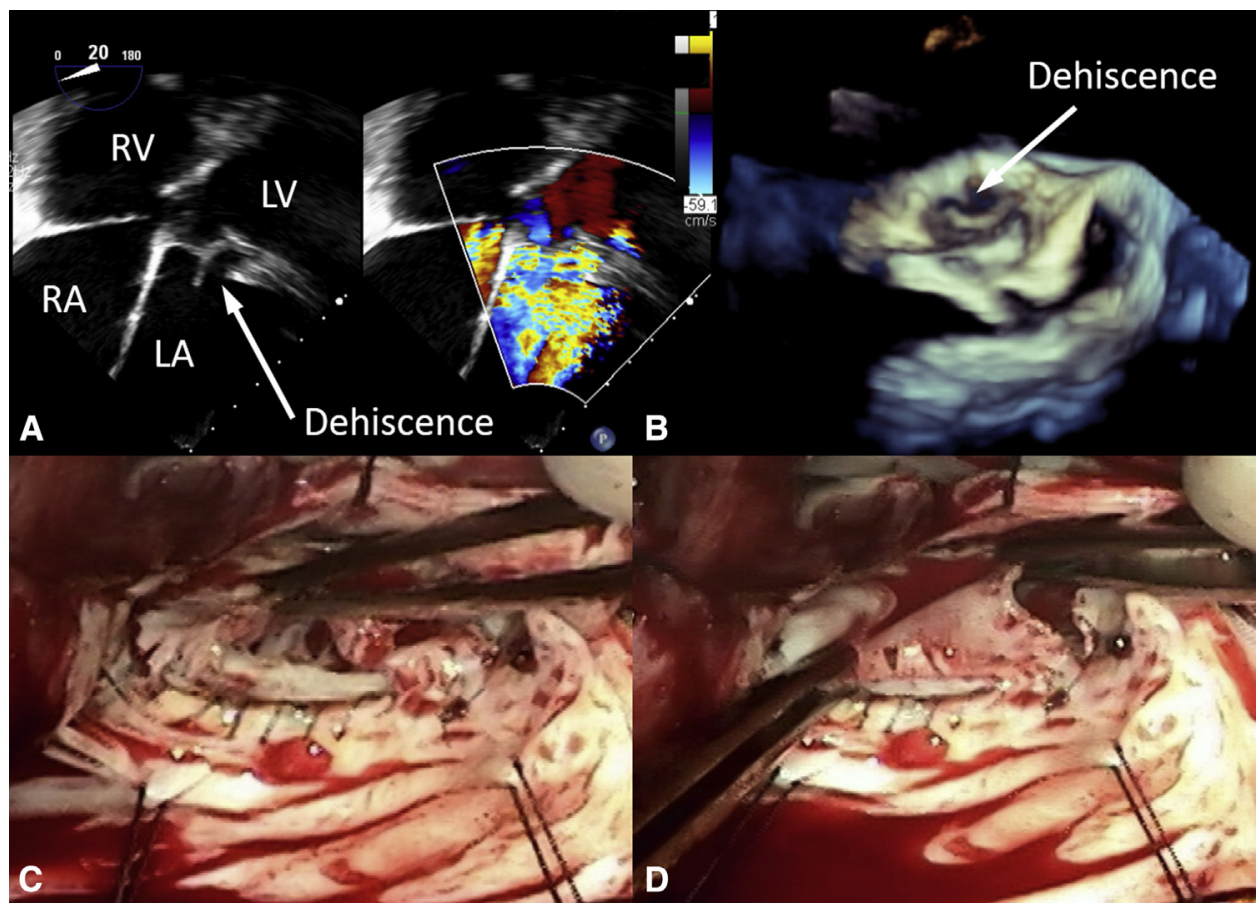

FIGURE 1. A, Color Doppler echocardiogram showing mural leaflet dehiscence (arrow) and severe valvular regurgitation. $R V$, Right ventricle; $L V$, left ventricle; $R A$, right atrium; $L A$, left atrium. B, 3-dimensional echocardiogram showing disruption of the mural leaflet at the annulus. Arrow indicates dehiscence. $\mathrm{C}$ and $\mathrm{D}$, Leaflet patch at the site of dehiscence from the annulus. Note the cuts or tears in the patch where sutures pulled through.

factor of $(1.6 \mathrm{~N} / \mathrm{mm}) /(1.6 \mathrm{~N} / \mathrm{mm})=1.0$, which corresponds to no margin for safety and is below the range of 2 to 10 commonly seen for both man-made and natural structures. ${ }^{3}$ In our experience, acute failure of a valve augmentation patch as seen in the case reported here is rare, suggesting that surgeons are generally quite good at producing repairs of adequate strength. Explicit knowledge of suture retention

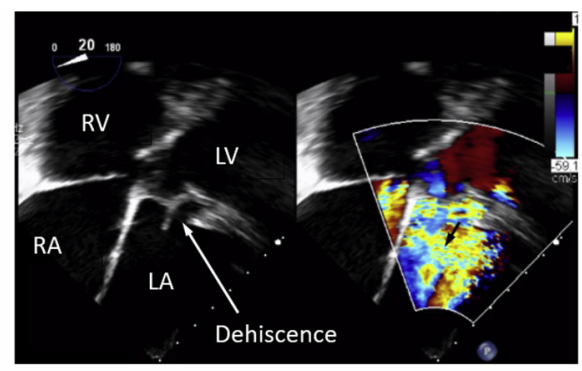

Preoperative transesophageal echocardiogram showed severe mitral regurgitation through the dehisced mural leaflet. LA, LV, RA, and RV denote left atrium, left ventricle, right atrium, and right ventricle, respectively. Black arrow depicts regurgitant jet.

VIDEO 1. In this video, we summarize the patient's history and presentation then show preoperative echocardiographic (echo) and intraoperative video evidence of severe left-sided atrioventricular valve regurgitation and patch dehiscence. $R V$, Right ventricle; $L V$, left ventricle; $R A$, right atrium; $L A$, left atrium; $3 D, 3$-dimensional. Video available at: https://www.jtcvs.org/article/S0022-5223(18)31240-6/fulltext. strength and a true worst-case estimate of transvalvular pressure, however, are necessary to design an adequate repair with confidence. Our results suggest several possibilities for raising the safety factor to ensure against patch dehiscence. Restricting strenuous isometric exercise could lower the peak expected load by one-half or more, increasing the safety factor to at least 2.0. Modifications to the surgical technique could produce a similar effect, however, without requiring exercise restriction. For example, orienting the patch with attention to the material direction could increase the safety factor to 1.7 , whereas reducing suture spacing to $1 \mathrm{~mm}$ could independently raise it to 2.0. Other factors known to affect suture line strength

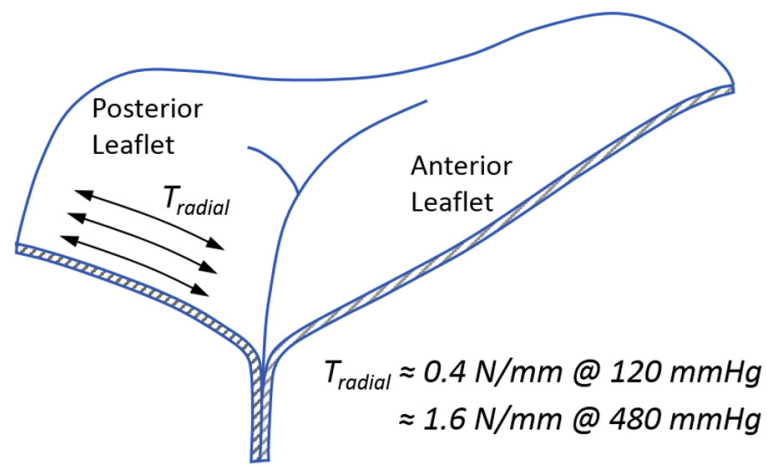

FIGURE 2. Leaflet load in the radial direction is expressed here as a membrane tension, $T_{\text {radial }}$, for both normal and elevated systolic pressures. 


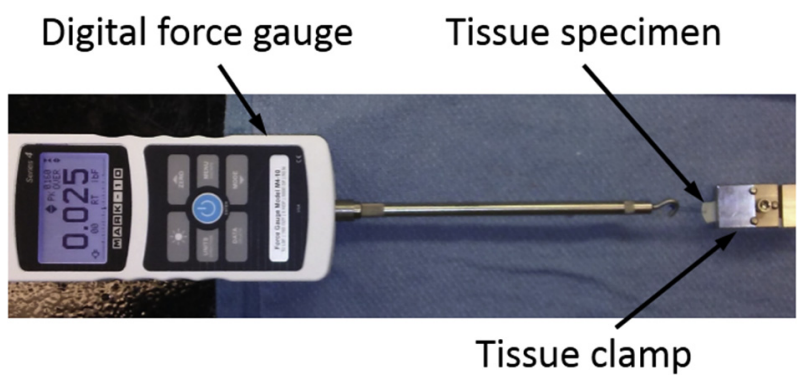

FIGURE 3. Suture retention tests use a digital force gauge connected to a loop of suture through the specimen. The opposite edge of the specimen is secured with a tissue clamp.

include the size of the suture material, the depth of the suture bite, and incorporation of suture line reinforcement material, although we did not evaluate these factors in this

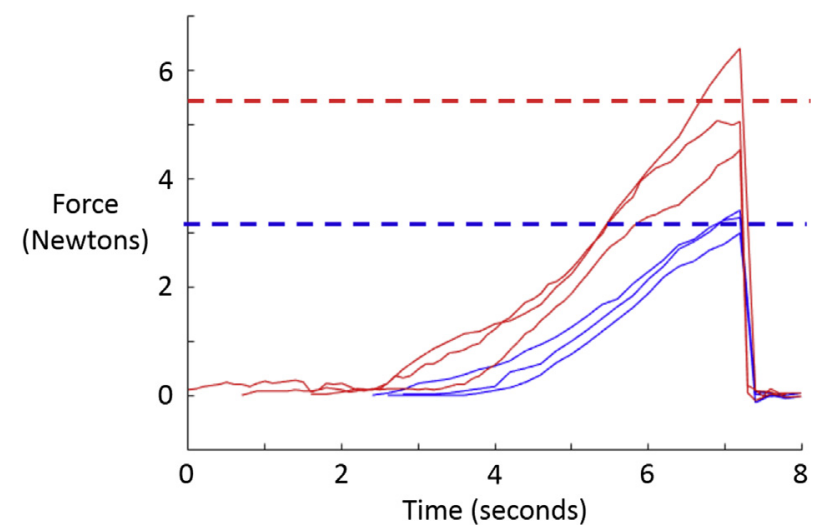

FIGURE 4. Results of suture retention tests with 5-0 Prolene suture (Ethicon, Inc, Somerville, NJ) in PhotoFix pericardium (CryoLife Inc, Kennesaw, Ga). Red curves correspond to tests in which the suture load was applied in the first principal material direction; blue curves correspond to tests in which suture load was applied in the second principal material direction. The steep fall in force corresponds to the suture tearing through the edge of the tissue. Red and blue dashed lines indicate the mean suture retention force in the corresponding material directions.

\section{Suture pull-out force $\approx 3.2 \mathrm{~N} /$ suture}

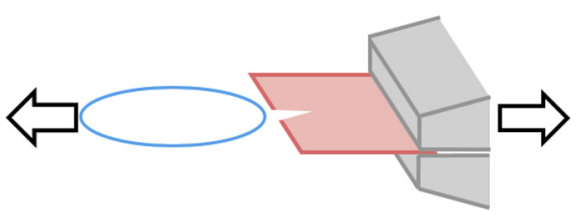

Ultimate strength of suture line

$$
=\frac{\text { Pull-out force }}{\text { Suture spacing }} \approx \frac{3.2 \mathrm{~N} / \text { suture }}{2 \mathrm{~mm} / \mathrm{suture}} \approx 1.6 \mathrm{~N} / \mathrm{mm}
$$

FIGURE 5. Suture line strength is calculated by dividing the suture pull-out force by the suture spacing.

study. We also did not consider the effect of changes to the integrity of the patch with time, nor did we compare suture retention strength in different patch materials, although we think that such a study is necessary. Finally, substantially increasing the size of a leaflet by patch augmentation can result in increased loads at the suture lines. Although it is difficult to precisely predict the magnitude of this effect, it is reasonable to estimate that leaflet stress increases in proportion to the increase in chamber size (Law of Laplace), and we could further scale the estimate for maximum expected load to account for this effect. Despite these limitations, we are hopeful that by adopting a quantitative approach to estimating maximum expected loads and suture line strength, we can avert similar premature failures of surgically repaired valves.

\section{References}

1. MacDougall JD, Tuxen D, Sale DG, Moroz JR, Sutton JR. Arterial blood pressure response to heavy resistance exercise. J Appl Physiol (1985). 1985;58:785-90.

2. Lee CH, Amini R, Gorman RC, Gorman JH III, Sacks MS. An inverse modeling approach for stress estimation in mitral valve anterior leaflet valvuloplasty for in-vivo valvular biomaterial assessment. J Biomech. 2014;47: 2055-63.

3. Diamond J. Quantitative evolutionary design. J Physiol. 2002;542(Pt 2):337-45. 


\section{APPENDIX. SUPPLEMENTARY MATERIAL Estimation of Loads in the Reconstructed Atrioventricular Valve}

Loads within tissue are represented as stress, with units of force per cross-sectional area of tissue. Although stresses in a valve leaflet cannot be measured directly, they can be estimated using computational modeling methods. Leaflet stresses at peak systole are, in general, different in different parts of the valve, and at a given location, leaflet stress can also vary in different directions. For this analysis, we are concerned with stress in the mural leaflet of the left-sided (systemic) atrioventricular valve. Specifically, we are concerned with leaflet stress in the radial direction (Figure 2) - that is, the direction perpendicular to the annulus-because this corresponds to the direction of the loads that resulted in the suture line dehiscence. At normal systolic pressures, peak stress in this region, in the radial direction, is estimated as follows ${ }^{2}: 400 \mathrm{kPa}=400,000 \mathrm{~N} / \mathrm{m}^{2}=0.4 \mathrm{~N} /$ $\mathrm{mm}^{2}$. For this analysis, it is more convenient to express leaflet loads as membrane tension, which is the force per length acting on a cross-section of leaflet. Assuming a leaflet thickness of $1 \mathrm{~mm}$, leaflet stress can be converted to membrane tension as follows: $0.4 \mathrm{~N} / \mathrm{mm}^{2} \times 1 \mathrm{~mm}=$ $0.4 \mathrm{~N} / \mathrm{mm}$.

During weight lifting, systolic pressure can increase by a factor of $4 .{ }^{1}$ Membrane tension is proportional to transvalvular pressure, so, assuming this worst-case scenario for pressure, a 4-fold increase in pressure would produce a 4fold increase in peak expected membrane tension (Figure 2): $0.4 \mathrm{~N} / \mathrm{mm} \times 4=1.6 \mathrm{~N} / \mathrm{mm}$.

\section{Estimation of Suture Line Strength}

We evaluated suture retention strength with PhotoFix pericardium (CryoLife Inc, Kennesaw, Ga) and 5-0 Prolene suture (Ethicon, Inc, Somerville, NJ), the same materials used in the valve repair. Pericardium is typically characterized by a principal material direction-that is, a direction in the tissue in which elongation under tension is at a minimum. We refer to this as the first principal material direction. In the direction perpendicular, elongation is a maximum, and we refer to this as the second principal material direction. Because this material anisotropy could result in direction-dependent suture retention capacity, we tested suture retention strength in both the first and second principal material directions. In 6 square tissue specimens, $10 \mathrm{~mm}$ on a side, a suture was placed $1.0 \mathrm{~mm}$ from an edge and tied to form a loop. The loop was placed around the hook of a digital force gauge (model M4; Mark-10 Corporation, Copiague, NY), and the opposite edge of the specimen was secured with a tissue clamp (CellScale Biomaterials Testing, Waterloo, Ontario, Canada), as shown in Figure 3. Tension was applied at a rate of approximately $1 \mathrm{~N} / \mathrm{s}$ until the suture pulled through the edge of the specimen. Three specimens were tested in each of the 2 principal material directions. Suture pullout force averaged $5.4 \pm 1.0 \mathrm{~N}$ and $3.2 \pm 0.2 \mathrm{~N}$ in the first and second principal material directions, respectively (Figure 4). The strength of the suture line can be expressed as membrane tension by dividing the suture pullout force by the suture spacing (Figure 5). For the weaker material direction (the second principal material direction) and a suture spacing of $2 \mathrm{~mm}$, the suture line strength is estimated as follows: $(3.2 \mathrm{~N} /$ suture $) /(2 \mathrm{~mm} /$ suture $)=1.6 \mathrm{~N} / \mathrm{mm}$. 